$7 p$ ip

$\approx \ldots$

$0163-12595$

CodE -1 NASA TND-1685

(1961)

TECHNICAL NOTE

D-1685

\title{
THE ORIGIN OF ATMOSPHERIC XENON
}

\author{
A. G. W. Cameron \\ Goddard Institute for Space Studies \\ New York, New York
}

NATIONAL AERONAUTICS AND SPACE ADMINISTRATION WASHINGTON

February 1963 


$$
c^{e^{\prime}}
$$

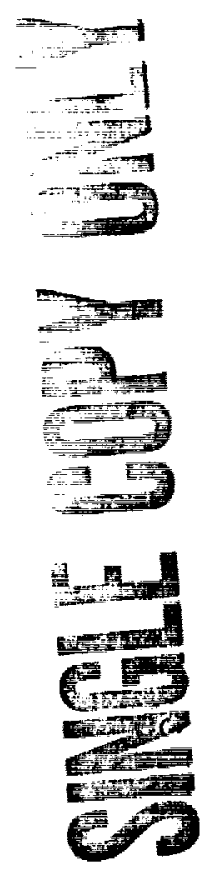




\title{
THE ORIGIN OF ATMOSPHERIC XENON
}

\author{
by \\ A. G. W. Cameron \\ Goddard Institute for Space Studies
}

\begin{abstract}
SUMMARY
Some of the differences in the isotopic composition of atmospheric and meteoritic xenon can be attributed to irradiation of solar xenon by neutrons during the deuterium burning phase of solar contraction, which was followed by accretion of the irradiated xenon into the atmosphere. This problem is examined here more carefully in view of a new measurement which has been made of the reaction rate for the capture of protons by deuterium. It is concluded that, apart from the xenon produced by the decay of extinct radioactivities, most of the atmospheric xenon was in the sun during the deuterium burning phase.
\end{abstract}





\section{CONTENTS}

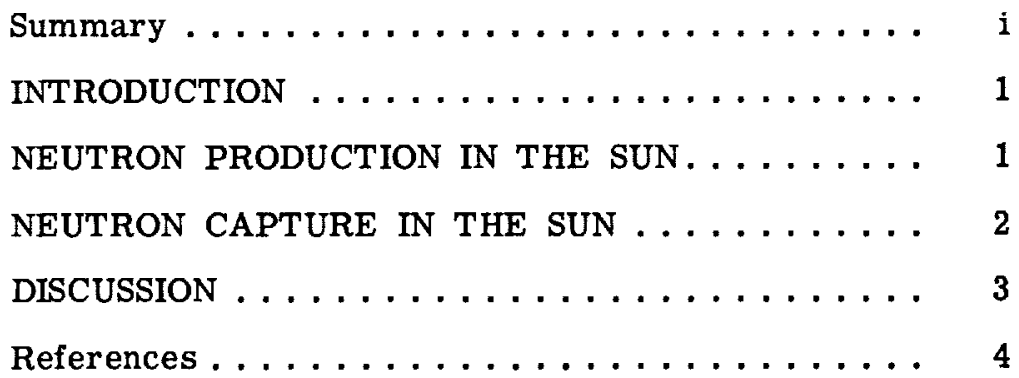





\title{
THE ORIGIN OF ATMOSPHERIC XENON
}

\author{
by \\ A. G. W. Cameron \\ Goddard Institute for Space Studies
}

\section{INTRODUCTION}

In a recent article by the author the differences between the isotopic composition of atmospheric and meteoritic xenon were analyzed (Reference 1). The differences were attributed to three causes: (1) the $\mathrm{Xe}^{129}$ decay product of the extinct radioactivity $\mathrm{I}^{129}$; (2) the fission products of the extinct radioactivity $\mathrm{Pu}^{244}$; and (3) the composition changes produced by neutron capture in one of the sources of atmospheric xenon. A crude quantitative analysis of the third cause led to the conclusion that the neutron capture had occurred during the deuterium burning phase of solar contraction, and that about $1 / 3$ of the atmospheric xenon had once resided in the post-deuterium-burning sun.

Recently, Griffiths, Lal, and Scorfe (private communication from G. M. Griffiths to H. Reeves) have redetermined the reaction rate at low energies for the $\mathrm{D}^{2}(\mathrm{p}, \gamma) \mathrm{He}^{3}$ reaction. This competes with the $D^{2}(d, n) H^{3}$ and $D^{2}(d, p) H^{3}$ reactions in the deuterium burning process. They have found that the $\mathrm{D}^{2}(\mathrm{p}, \gamma) \mathrm{He}^{3}$ reaction rate is greater than that given by Salpeter (Reference 2) by a factor 2.4. Dr. Griffiths states that the error in the new value may be as large as 50 percent, but is probably somewhat smaller.

In view of this change, the question of the solar origin of some atmospheric xenon has been examined more carefully, and this paper reports the results.

\section{NEUTRON PRODUCTION IN THE SUN}

Equation 1 of Reference 1 gives the ratio of probabilities for the reaction of a deuteron with a deuteron relative to that of a deuteron with a proton. Let us divide this ratio by the factor 2 in order to count only those deuteron-deuteron reactions that produce neutrons, and also let us utilize the new reaction rate of Griffiths, Lal, and Scorfe. The modified ratio then becomes:

$$
\frac{P_{n}}{P_{p}}=1.24 \times 10^{5}\left(\frac{x_{D}}{x_{H}}\right) \exp \left(-\frac{5.4}{T_{6}^{\frac{1}{3}}}\right),
$$

where $P_{n}$ and $P_{p}$ are the reaction rates for producing neutrons and absorbing protons, $x_{D}$ and $x_{H}$ 
are the concentrations by weight of deuterium and hydrogen, and $T_{6}$ is the temperature in units of $10^{6}{ }^{\circ} \mathrm{K}\left(\mathrm{T}_{6}=\mathrm{T} \times 10^{6}{ }^{\circ} \mathrm{K}\right)$.

It has been pointed out recently by Hayashi that the contracting sun must have been fully convective until it approached quite near the main sequence configuration (Reference 3 ). This has been confirmed by Ezer and Cameron, who found that deuterium burning took place during the fully convective phase and lasted only about $3 \times 10^{5}$ years because of the relatively high luminosity of the sun at the time (Reference 4). Thus we can be assured that all the material in the sun was subjected to the neutron flux associated with deuterium burning, and that this occurred early in the history of the solar system.

With the previous assumptions of Cameron that $\mathrm{x}_{\mathrm{D}} / \mathrm{x}_{\mathrm{H}}=3 \times 10^{-4}$ initially and that $\mathrm{T}_{6}=0.8$ (Reference 1), it follows from Equation 1 of this paper that $P_{n} / P_{p}=0.111$. Hence, allowing for the fact that this is twice the average rate of neutron production, we find that there will be 0.046 neutron produced per initial deuteron present in the sun.

\section{NEUTRON CAPTURE IN THE SUN}

The neutrons produced during deuterium burning become thermalized and then are captured by surrounding nuclei in proportion to the capture cross sections. We are particularly interested in finding the number of neutrons captured by $\mathrm{I}^{127}$ to form, after beta decay, an excess abundance of $\mathrm{Xe}^{128}$.

The capture cross section in $I^{127}$ must be computed by summing the contributions from all effective resonances of the compound nucleus. Each resonance makes a contribution to the average cross section of

$$
\left\langle\sigma\left(\mathrm{E}_{\mathrm{r}}\right)\right\rangle=555 \frac{\mathrm{g} \Gamma_{\mathrm{n}} \Gamma_{\gamma}}{\mathrm{T}_{6} \Gamma} \exp \left(\frac{-0.01161 \mathrm{E}_{\mathrm{r}}}{\mathrm{T}_{6}}\right)
$$

where $E_{r}$ is the resonance energy in ev; $\mathrm{g}=(2 \mathrm{~J}+1) / 2(2 \mathrm{I}+1)$ is a statistical factor, $\mathrm{J}$ and $\mathrm{I}$ being the compound and initial spins of the nucleus under consideration; and $\Gamma_{n}, \Gamma_{\gamma}$, and $\Gamma$ are the neutron, radiation, and total widths of the resonance in ev. The most complete data for the resonances in the compound nucleus $\mathrm{I}^{128}$ appear to be those of Desjardins, et al. (Reference 5), as quoted in the Nuclear Data Tables. The average cross section was computed from these data with the assumptions $\mathrm{g}=0.5$ and $\Gamma_{\gamma}=0.1$ if not directly measured. The result is $\langle\sigma\rangle=25$ barns. This is probably correct to about ten percent. Most of the neutrons produced will react with hydrogen and $\mathrm{He}^{3}$. The capture in any other nucleus can be computed as a small perturbation on the neutron elimination by these two nuclei. The situation is slightly complicated by the fact that $\mathrm{He}^{3}$ is produced by the deuterium burning process.

Both $\mathrm{H}^{1}$ and $\mathrm{He}^{3}$ have neutron elimination cross sections varying as $1 / \mathrm{v}$, where $\mathrm{v}$ is the neutron velocity. At $\mathrm{T}_{6}=0.8$, the cross section averaged over the Maxwell distribution of velocities is equal to the actual cross section at $87.6 \mathrm{ev}$. Hence for $\mathrm{H}^{1},\langle\sigma\rangle=0.0056 \mathrm{barns}$, and for $\mathrm{He}^{3}$, 
$\langle\sigma\rangle=91$ barns. Neutron capture in $\mathrm{H}^{1}$ produces additional deuterium, but we shall neglect the second order production of additional neutrons arising from this fact. The neutron interaction with $\mathrm{He}^{3}$ gives the reaction $\mathrm{He}^{3}(n, p) \mathrm{H}^{3}$, but the tritium decays back to $\mathrm{He}^{3}$ rapidly, compared to the deuterium burning time of the contracting sun. Hence we can neglect any depletion of $\mathrm{He}^{3}$ due to neutron capture.

There appears to be remarkably little variation in the ratio $\mathrm{He}^{3} / \mathrm{He}^{4}$ in primordial gases extracted from meteorites (private communication from P. Signer). The average number ratio of these nuclei is approximately $3 \times 10^{-4}$. If we assume a number ratio of $\mathrm{He}^{4} / \mathrm{H}^{1}$ equal to 0.156 , then we obtain a primordial number ratio of $\mathrm{He}^{3} / \mathrm{H}^{1}$ equal to $4.7 \times 10^{-5}$. Multiplying this ratio by $91 / 0.0056$, the ratio of neutron cross sections, we see that the primordial $\mathrm{He}^{3}$ is 0.76 times as effective as $\mathrm{H}^{1}$ for eliminating neutrons. During deuterium burning 0.954 of the deuterium was converted to $\mathrm{He}^{3}$, and hence, with an assumed number ratio $\mathrm{D}^{2} / \mathrm{H}^{1}$ equal to $1.5 \times 10^{-4}$, the $\mathrm{He}^{3}$ produced is 2.32 times as effective as $\mathrm{H}^{1}$ in eliminating neutrons.

The number of neutrons produced per hydrogen atom is $0.046 \times 1.5 \times 10^{-4}=6.9 \times 10^{-5}$. Hence the number of neutrons captured by any other nucleus with average capture cross section $\langle\sigma\rangle$ is approximately

$$
\mathrm{f}=\frac{6.9 \times 10^{-6}\langle\sigma\rangle}{0.0056} \int_{0}^{1} \frac{\mathrm{dx}}{1+0.76+2.32 \mathrm{x}}=4.5 \times 10^{-4}\langle\sigma\rangle
$$

In the case of particular interest here, $\mathrm{I}^{127}, \mathrm{f}=0.0113$.

\section{DISCUSSION}

In Reference 1 the conclusions regarding the solar origin of some atmospheric xenon were based on Table 3 of that paper, in which it was found that, relative to $\mathrm{Xe}^{124}$ and $\mathrm{X}^{126}$, which should be only negligibly depleted by neutron capture, $\mathrm{Xe}^{128}$ was overabundant in the atmosphere by $15 \pm 2$ percent. This is to be attributed to neutron capture in $I^{127}$ in the sun.

In the preceding section, we have found that about 0.0113 of the $\mathrm{I}^{127}$ should be destroyed by neutron capture in the sun. Relative to the normalization used in Table 3 of Reference 1 , the abundance of $\mathrm{I}^{127}$ is approximately 8. Hence the excess $\mathrm{Xe}^{128}$ that should be formed during deuterium burning in solar material is $8 \times 0.0113$, or 9 percent.

A comparison of these two numbers seems to indicate that atmospheric xenon contains more excess $\mathrm{Xe}^{128}$ than can be accounted for by neutron capture in pure solar material. However, if we take the full 50 percent error allowable in the new determination of the $D^{2}(p, \gamma) \mathrm{He}^{3}$ reaction rate, then it appears that the two numbers barely agree within the overlap of their errors.

Thus, this interpretation of the xenon isotopic anomalies leads to the conclusion that the majority of all xenon in the atmosphere that does not result from the decay of extinct radioactivities was once part of the sun during the deuterium burning phase. There is no evidence for any xenon surviving in 
the atmosphere from a primitive protoplanet in which the earth may be thought to have formed; such residual xenon could be at most a small fraction of the atmospheric xenon. Hence the majority of the noble gases in the atmosphere, except for helium and $\mathrm{A}^{40}$, have probably accreted from the solar wind (Reference 1). Although it was argued in Reference 1 that the data of Krummenacher, Merrihue, et al. (Reference 6 ) on the isotopic composition of krypton were slightly inconsistent with this conclusion, the uncertainty in the solar abundance ratio of bromine and krypton renders the argument inconclusive. Further work on krypton would be desirable.

If the noble gases can accrete from the solar wind, what about hydrogen? Could the deuterium to hydrogen ratio in terrestrial waters have been changed relative to the primordial ratio? Boato measured this ratio for several carbonaceous chondrites, and found $\mathrm{D}^{2} / \mathrm{H}^{1}$ ratios as high as $2 \times 10^{-4}$ (Reference 7). Thus it is quite possible that the primordial sun may have had considerably more deuterium than has so far been assumed in this paper.

If the calculations of this paper are repeated with $\mathrm{x}_{\mathrm{D}} / \mathrm{x}_{\mathrm{H}}=4 \times 10^{-4}$, then we obtain $\mathrm{f}=0.0125$ for ${ }^{127}$. Hence on the normalization of Table 3 of Reference 1 we would expect a 10 percent excess $\mathrm{Xe}^{128}$ abundance in solar material. This gives a slight improvement in the agreement of the predicted and observed values. Clearly it is desirable to investigate the deuterium content of carbonaceous chondrites more thoroughly. The major source of error in the predicted value remains the $\mathrm{D}^{2}(\mathrm{p}, \gamma) \mathrm{He}^{3}$ reaction rate, and it is particularly desirable to improve that measurement further.

\section{REFERENCES}

1. Cameron, A. G. W., "The Formation of the Sun and Planets," Icarus 1(1):13-69, May 1962.

2. Salpeter, E. E., "Nuclear Reactions in Stars. II. Protons on Light Nuclei," Phys. Rev. 97(5):12371244, March 1, 1955.

3. Hayashi, C., "Stellar Evolution in Early Phases of Gravitational Contraction," Publ. Astronom. Soc. Japan 13(4):450-452, 1961.

4. Ezer, D., and Cameron, A. G. W., papers presented at the 111th meeting of the Amer. Astronom. Soc., Yale Univ., New Haven, Conn., August 1962: "High-Luminosity, Fully Convective Contracting Sun. I," Astronom. J. 67(9):575, November 1962 (abstract); "High-Luminosity, Fully Convective Contracting Sun. II," Astronom. J. 67(9):572, November 1962 (abstract).

5. Desjardins, S., Havens, w. W., Jr., et al., "Neutron Resonances in Th, I, Br," Bull. Am. Phys, Soc. Ser. 2, 5(4):295, April 25, 1960 (abstract).

6. Krummenacher, D., Merrihue, C. M., et al., preprint, 1961.

7. Boato, G., "The Primeval Cosmic Abundance of Deuterium," Phys. Rev. 93(3):640-641, February 1, 1954. 